\title{
Silenced $\mathrm{X}$ chromosome genes reawaken in new mouse model
}

\section{BY HOLLY BARKER}

3 MARCH 2022

A new mouse model may accelerate the discovery of gene-activating therapies to treat Rett syndrome, a condition related to autism, its makers say.

Rett syndrome arises from mutations in MECP2, a gene on the $X$ chromosome. Such mutations are usually fatal shortly after birth in boys, who have only one $X$ chromosome and thus no backup copy of the gene. Girls have two X chromosomes, but a process called random X inactivation silences one of them in about half of their cells. As a result, their backup copy of MECP2 can't compensate for a mutated one - leading to the syndrome's core traits, which include intellectual disability and seizures.

Researchers have been attempting to awaken the silenced copy to treat the condition. To test their strategies, they typically couple the MECP2 gene to DNA that encodes a fluorescent jellyfish protein. However, the fluorescent signal is weak and may not detect subtle changes in gene activation. Alternatively, the gene is fused with luciferase -- the bioluminescent enzyme found in fireflies. This is brighter and more sensitive, but the bulky protein can disrupt the function of the MECP2 protein.

In the new mouse model, researchers fused the MECP2 gene to DNA that encodes a nanosized version of luciferase that, despite being more compact, emits 100 times as much light as the original. It can detect smaller changes in MECP2 reactivation and is less likely to disrupt the function of the MECP2 protein, says study investigator Antonio Bedalov, professor of medicine at the Fred Hutchinson Cancer Research Center in Seattle, Washington. The mice also express a red fluorescent protein when the bioluminescent version of MECP2 is active; that tag enables the researchers to track which cells carry the modified gene.

Bedalov and his colleagues bred female mice to harbor the bioluminescent version of MECP2 on their silenced copy of the $\mathrm{X}$ chromosome. The team then extracted neural stem cells from the mice, 


\section{Spectrum | Autism Research News}

https://www.spectrumnews.org

grew them in culture dishes and treated them with four compounds known to reactivate MECP2.

Researchers typically use mouse fibroblasts in such experiments, but neural stem cells are more relevant to Rett syndrome, says study investigator Hegias Mira-Bontenbal, associate professor at Erasmus Medical Centre in Rotterdam, the Netherlands.

Only one of the compounds Mira-Bontenbal and his colleagues used, called 5-azacytidine, successfully lit up the neural stem cells, signaling MECP2 reactivation. The drug works by blocking an enzyme from adding to DNA repressive tags that turn genes off. Combining the drug with short nucleotide strands that inhibit a molecule involved in silencing the $\mathrm{X}$ chromosome boosted MECP2 expression further.

To determine how many other genes along the $\mathrm{X}$ chromosome were also reactivated by the treatment, the researchers took advantage of genetic variation between the two $X$ chromosomes the bred mice inherited one $X$ from an Algerian mouse strain called Cast/EiJ and the other $X$ from a standard C57BL/6 laboratory mouse. These two sets of mice are as genetically similar to each other as people are to chimpanzees, Bedalov says.

Sequencing the active genes from the $X$ chromosome and searching for stretches of nucleotides unique to the Algerian mice revealed reactivation of 85 other genes. The study was published in February in Stem Cell Reports.

Other groups are already using the mouse-derived cell lines, Mira-Bontenbal says, to investigate how different MECP2 mutations affect how well the protein works and how quickly it is degraded, shedding light on how the condition progresses at the cellular level.

Mira-Bontenbal says that he and his colleagues aim to test hundreds of chemical compounds to assess their ability to reactivate MECP2, an otherwise labor-intensive process. The team is creating a similar nano-luciferase system in human cells to see whether pharmacological therapies translate to people.

Meanwhile, Bedalov and his colleagues are using a modified version of CRISPR to remove the repressive tags on MECP2, in the hope of reactivating MECP2 without switching on other genes. They plan to test the effectiveness of that method using the mouse model, Bedalov says.

\section{Cite this article: https://doi.org/10.53053/XHXT2388}

